\title{
基于改进的速度障碍法的有人/无人机 协同系统三维实时避障方法
}

\author{
李樾 $^{1}$, 韩维 ${ }^{1}$, 陈清阳 ${ }^{2}$, 张勇 $^{1}$
}

(1.海军航空大学 航空基础学院, 山东 烟台 264001；2. 国防科技大学 空天科学学院, 湖南 长沙 410073)

\begin{abstract}
摘 要: 为适应当前智能体自主化水平, 同时发挥多智能体在空战中的优势, 有人/无人机协同系统的 作战形式逐渐成为研究热点。针对协同系统在三维空间内的实时避障问题, 首先, 在传统速度障碍法 的基础上,建立三维机动障碍的模型; 其次通过设定系统遇障时的通行法则，选择飞行模式，进而确定 最佳的避障平面及航迹; 最后,通过 3 组仿真分别校验避障平面的差异性、单机躲避机动性障碍的可 行性以及协同系统整体避障的有效性。仿真结果表明,该方法能安全快速地实现有人/无人机协同系 统对三维机动障碍的躲避。
\end{abstract}

关 键 词:有人/无人机; 速度障碍法; 三维空间; 避障平面; 实时避障

中图分类号: V249.1

文献标志码: A

有人无人机协同作战是指在信息化、网络化及 体系对抗环境下, 有人机与无人机联合编队实施协 同攻击的作战方式 ${ }^{[1]}$ 。该作战形式是潜在的第六 代穿透型战机发展方向之一 ${ }^{[2]}$, 美国于 2015 年提出 “忠诚僚机” 计划 ${ }^{[3]}$, 并于 2017 年利用有人战斗机 和由 F-16 改装的无人机验证了系统对地打击的效 能 ${ }^{[4]}$ 。为提高有人/无人机系统中有人机的生存能 力和作战效能, 进而保证整个系统的飞行安全, 研究 三维空间内的实时避障技术是十分重要的。

交互避障法是目前最常用的一种在线避障的方 法, 其主要包括势能域法、几何引导法。几何引导法 又包括威胁雉法 ${ }^{[5-6]}$ 、速度障碍物法 ${ }^{[7-8]}$ 和几何微分 法 $^{[9]}$ 等。由于速度障碍物法更为适用于复杂环境 下的多障碍物避障, 且对计算要求不高, 因此该方法 受到了广泛关注。

文献 $[10]$ 根据机器人和障碍物的实时状态量, 采取传统的速度障碍法对动态环境下的机器人进行 航迹规划; 文献 [11] 改进了速度障碍法, 着重考虑 了“潜在危险障碍” 的影响和多障碍避障的问题; 文 献[12]提出了二维平面内的选择速度障碍法, 不足 之处在于未考虑外部障碍对系统中飞机的影响; 文
文章编号: 1000-2758(2020)02-0309-10

献 [13-14]分别将速度障碍法运用到二维空间内多 机同构系统躲避机动障碍和三维空间内单机躲避非 机动障碍中。

综上, 将现有的速度障碍法运用到有人无人机 协同系统中仍有一定的难度。本文以传统速度障碍 法为基础, 提出一种有人无人机协同系统的三维实 时避障方法, 其思想是将三维空间离散成若干避障 平面, 通过设定系统遭遇机动障碍时的通行法则, 选 择飞行模式,进而确定最佳的避障平面以实现有人 机与无人机的协同避障。

\section{1 三维速度障碍法建模}

\section{1 传统速度障碍法}

传统速度障碍 (velocity ostacle, VO) 法建立在二 维空间内, 将飞机视为一质点, 根据已知的飞机和障 碍物 $S$ 之间的相对大小关系对 $S$ 进行“膨化” 处理， 利用半径 $r$ 作为表征 $S$ 膨化量的指标。

设飞机与 $S$ 的飞行速度分别为 $\boldsymbol{V}_{o}$ 和 $\boldsymbol{V}_{s}$, 位置向 量分别为 $\boldsymbol{X}_{o}$ 和 $\boldsymbol{X}_{s}$, 依据威胁雉理论 ${ }^{[5]}$, 当飞机与 $S$ 
的相对速度 $V_{R}$ 位于威胁雉内时, $S$ 被视为威胁障 碍。在此基础上, 沿 $\boldsymbol{V}_{s}$ 方向移动威胁雉, 得到速度 障碍雉 $\mathrm{VO}$, 其原理图如图 1 所示。图中, 下标 $i, j=$ $1,2,3 \cdots$, 分别表示飞机和障碍物的标号; $\boldsymbol{D}_{s}$ 为飞机 与 $S$ 的矢量距离 (绝对值为 $d_{s}$ ), 该量可实时获取; 由 于锥的高度不定, 因而用波浪线的形式表示其不断 延伸的底边, 后图同理。为成功避障, 应保证 $\boldsymbol{V}_{o}$ 矢 量的终端位于 $\mathrm{VO}$ 之外, 即 $\boldsymbol{V}_{o} \notin \mathrm{VO}$ 。

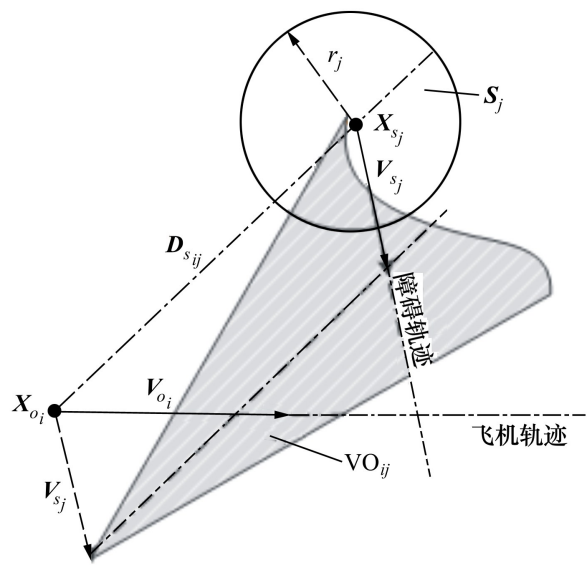

图 1 二维速度障碍雉原理图

\section{2 三维速度障碍法的参数设定}

下面将传统 VO法拓展到三维中。首先, 将 $S$ 由 平面圆转换为一个球体, 相比转换为柱体或雉体, 球 体可以较好地模拟飞机等机动性较强的动态障碍 物, 且对系统中不同来向的飞机具有同等的威胁。

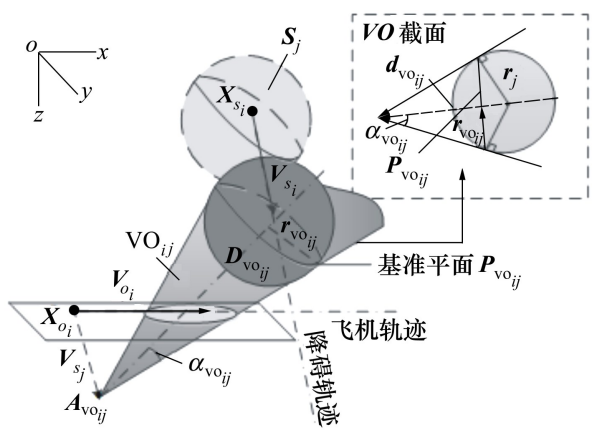

图 2 三维速度障碍雉原理图

其次, 确定构成三维 VO 的 3 个重要参数, 即雉 顶点位置向量 $\boldsymbol{A}_{\mathrm{vo}}$ 、雉的半顶角 $\alpha_{\mathrm{vo}}$ 、飞机到基准平面 $P_{\mathrm{vo}}$ 的矢量距离 $\boldsymbol{D}_{\mathrm{vo}}$ (绝对值为 $d_{\mathrm{vo}}$ )。具体模型如图 2 所示。图中已出现的变量的物理含义与图 1 保持一 致; 坐标系 $o-x y z$ 表示飞机 $i$ 的机体坐标系; $P_{\mathrm{vo}_{i j}}$ 是球 $S_{j}$ 与 $\mathrm{VO}_{i j}$ 相内切的圆截面, 半径记为 $r_{\mathrm{vo}_{i j} \text { 。 }}$

结合可探测获取的 $S$ 相对于飞机的俯仰角 $\theta$ 、偏
航角 $\psi$, 可计算出上述参数,如 (1) 式所示

$$
\left\{\begin{array}{l}
d_{\mathrm{vo}}=\frac{d_{s}^{2}-r^{2}}{d_{s}}, d_{s}>r \\
r_{\mathrm{vo}}=r \frac{\sqrt{d_{s}^{2}-r^{2}}}{d_{s}} \\
\alpha_{\mathrm{vo}}=\arctan \left(\frac{r_{\mathrm{vo}}}{d_{\mathrm{vo}}}\right) \\
\boldsymbol{A}_{\mathrm{vo}}=\boldsymbol{V}_{s} \\
\boldsymbol{D}_{\mathrm{vo}}=\left[\begin{array}{l}
\cos \theta \cos \psi \\
\cos \theta \sin \psi \\
\sin \theta
\end{array}\right] d_{\mathrm{vo}}
\end{array}\right.
$$

通过比较 $\alpha_{\mathrm{vo}_{-} t}$ 与 $\alpha_{\mathrm{vo}}$ 的大小, 可判断飞机沿 $\boldsymbol{V}_{o}$ 飞行是否会与 $S$ 发生碰撞, 其中 $\alpha_{\mathrm{vo}_{-} t}$ 是 $\boldsymbol{V}_{R}$ 与 VO 轴 线所夹的角度。当下式成立时, 飞机需进行避障

$$
\left\{\begin{array}{l}
\boldsymbol{V}_{o} \in \mathrm{VO} \Leftarrow\left\{\cos \alpha_{\mathrm{vo}_{-} t}>\cos \alpha_{\mathrm{vo}}\right\}, \text { 其中 } \alpha_{\mathrm{vo}}<\frac{\pi}{2} \\
\cos \alpha_{\mathrm{vo}_{-} t}=\frac{\left[\boldsymbol{V}_{o}-\boldsymbol{A}_{\mathrm{vo}}\right] \cdot \boldsymbol{D}_{\mathrm{vo}}}{\left|\boldsymbol{V}_{o}-\boldsymbol{A}_{\mathrm{vo}}\right| d_{\mathrm{vo}}}
\end{array}\right.
$$

\section{3 障碍物机动性的影响}

飞机在飞行过程中探测到的动态障碍, 例如有 人操纵的敌机以及系统中突然改变航迹的无人机 等, 均具有一定机动能力, 如按照非机动避障, 则可 能发生碰撞。

设飞机所携的距离探测设备每隔 $\Delta t$ 的时间进 行一次测量, 在 $\Delta t$ 内, 具有机动性的障碍可能以 $\boldsymbol{V}_{s}$ 为基准发生机动 (考虑偏航和俯仰 2 个自由度), 机 动区间为 $[-\omega \cdot \Delta t, \omega \cdot \Delta t]$, 其中 $\omega$ 为其最大机动角 速度; 则以 $\boldsymbol{A}_{\mathrm{v}}$ 为球心, 可确定一个半径为 $r_{m}$ 的机动 球, 记作 $M_{\mathrm{vo}}$; 进而沿 $\boldsymbol{D}_{\mathrm{vo}}$ 向量的反方向移动 $\mathrm{VO}$, 直 到 $M_{\mathrm{vo}}$ 正好内切于新的 $\mathrm{VO}$, 记此时的 $\mathrm{VO}$ 为 $\mathrm{VO}^{+}$。

图 3 为 $\mathrm{VO}^{+}$在纵截面内生成的原理图, 图中 $\boldsymbol{A}_{\mathrm{v}_{i j}}^{\prime}$ 和 $\boldsymbol{A}_{\mathrm{v}_{\mathrm{v} i j}}^{\prime \prime}$ 为偏航自由度上的临界机动点。

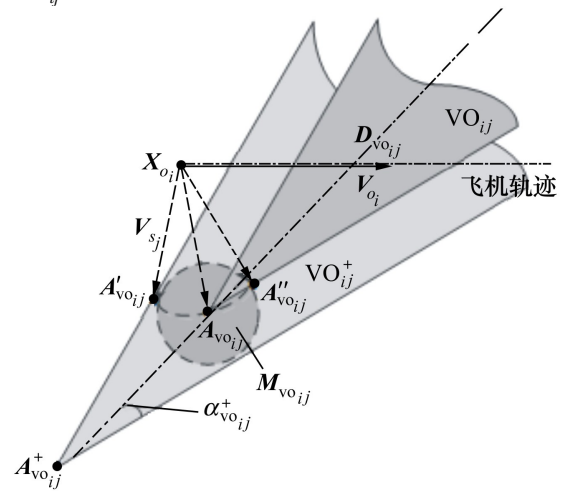

图 3 考虑障碍机动性的速度障碍雉 
与 $\mathrm{VO}$ 相同, $\mathrm{VO}^{+}$的构建也需要 1.2 节提到的 3 个参数, 而这些参数均可通过 VO 中已知的参数推 导出来, 无需增加新的未知变量, 因此该方法具有一 定的可行性。

利用相似三角形的原理, 可得出 $\mathrm{VO}^{+}$中的参数 $\alpha_{\mathrm{vo}}^{+}, \boldsymbol{A}_{\mathrm{vo}}^{+}$和 $\boldsymbol{D}_{\mathrm{vo}}^{+}$, 其计算如(3) 式所示

$$
\left\{\begin{array}{l}
\alpha_{\mathrm{vo}}^{+}=\alpha_{\mathrm{vo}} \\
\boldsymbol{A}_{\mathrm{vo}}^{+}=\boldsymbol{A}_{\mathrm{vo}}-r_{m} \frac{\boldsymbol{D}_{\mathrm{vo}}}{d_{\mathrm{vo}} \sin \alpha_{\mathrm{vo}}^{+}} \\
\boldsymbol{D}_{\mathrm{vo}}^{+}=\left[\begin{array}{l}
\cos \theta \cos \psi \\
\cos \theta \sin \psi \\
\sin \theta
\end{array}\right]\left(d_{\mathrm{vo}}+d_{\mathrm{vo}}^{+}\right)
\end{array}\right.
$$

式中, $r_{m}, d_{\mathrm{vo}}^{+}$的求解如 (4) 式所示。其原理是利用 半角公式求解 $\triangle X_{o} A_{\mathrm{vo}} A_{\mathrm{vo}}^{\prime}$ 半顶角的正弦值, 再利用 边角对应的几何关系求出所需变量。

$$
\left\{\begin{array}{l}
r_{m}=\left|\boldsymbol{V}_{s}\right| \sqrt{2(1-\cos (\omega \cdot \Delta t))} \\
d_{\mathrm{vo}}^{+}=\frac{r_{m}}{\sin \alpha_{\mathrm{vo}}^{+}}
\end{array}\right.
$$

至此, 考虑障碍物机动性的三维速度障碍法模 型已构建完毕,下文均以 $\mathrm{VO}^{+}$为模型进行研究。

\section{2 系统实时避障航迹的生成}

\section{1 避障平面的划分}

相对于二维算法, 三维实时避障更为复杂。如 果用解析法直接求解, 代数方程将十分繁琐, 甚至可 能得不到解析解, 当拓展到多机系统时这一问题将 更为显著。

针对该问题, 本文引人避障平面的概念。将三 维空间离散成若干平面, 利用这些平面截取 $\mathrm{VO}^{+}$获 得平面曲线,通过计算 $\boldsymbol{V}_{o}$ 与平面曲线的位置关系, 改变 $\boldsymbol{V}_{o}$ 的方向, 以达到避障的效果, 其中离散生成 的若干平面统称为避障平面。具体流程如下:

首先, 在避障飞机的机体坐标系下, 将平面 $x o y$ 绕 $x$ 轴旋转, 由右手定则确定旋转的正方向, 每旋转 角度 $\varphi_{0}$ 记录一个避障平面 $P_{a}$, 旋转区间为 $[0, \pi)$, 则共生成 $\pi / \varphi_{0}$ 个避障平面; 其次, 获取 $\mathrm{VO}^{+}$在避障 平面 $P_{a}$ 上的投影面 $\mathrm{VO}_{p}^{+}$, 得到 $\boldsymbol{V}_{o}$ 与 $\mathrm{VO}_{p}^{+}$中曲线的
位置关系; 最后, 根据一定的法则确定出最优避障平 面, 具体法则在 $2.2 \sim 2.3$ 节中讨论。

$\mathrm{VO}_{p}^{+}$中的曲线类型由基准平面 $P_{\mathrm{vo}}$ 与 $P_{a}$ 之间的 夹角 $\lambda_{a}$ 决定, 可通过计算 $\boldsymbol{D}_{\mathrm{vo}}$ 与 $P_{a}$ 法向量之间夹角 的方式求出, 其中 $P_{a}$ 法向量的求解用到了坐标转换 矩阵 $\boldsymbol{R}_{1}$, 具体如 (5) 式所示

$$
\left\{\begin{array}{l}
\lambda_{a}=\arccos \left(\frac{D_{v o}}{d_{v o}} \cdot R_{1} \cdot[0,0,1]^{\mathrm{T}}\right) \\
\boldsymbol{R}_{1}=\left[1,0,0 ; 0, \cos \varphi_{a}, \sin \varphi_{a} ; 0,-\sin \varphi_{a}, \cos \varphi_{a}\right] \\
\varphi_{a}=n_{a} \cdot \varphi_{o}
\end{array}\right.
$$

式中, $n_{a}$ 为 $P_{a}$ 旋转 $\varphi_{o}$ 角度的次数。当 $\alpha_{v o}^{+}<\pi / 2-$ $\lambda_{a}$ 时, $P_{a}$ 可穿过 $V O^{+}, V O_{p}^{+}$的曲线类型为椭圆; 而当 $\alpha_{v o}^{+} \geqslant \pi / 2-\lambda_{a}$ 时, $P_{a}$ 无法穿过 $V O^{+}, V O_{p}^{+}$的曲线类 型通常为双曲线,特殊情况下还可能为三角形。后 者的几何关系不适用于 VO 法的研究, 因此本文仅 讨论 $V O_{p}^{+}$的曲线类型为椭圆时的情况。

在 $V O^{+}$中, 设由 $\boldsymbol{A}_{v o}^{+}$起, 沿轴线延伸某一距离 $l\left(0<l \leqslant d_{v o}\right)$, 得到平行于 $P_{v o}$ 的平面 $P_{l \circ}$ 设该平面 绕轴线可旋转的角度为 $\beta(0 \leqslant \beta<2 \pi)$, 则在图 2 所 示的机体坐标系下, 通过空间内的坐标变换可得到 $V O^{+}$雉面上任意点的坐标 $\left[x_{v o}, y_{v o}, z_{v o}\right]$, 具体通过 (6) 式计算

$$
\left\{\begin{array}{l}
{\left[x_{v o}, y_{v o}, z_{v o}\right]=\boldsymbol{R}_{1} \cdot\left(\boldsymbol{R}_{3} \cdot \boldsymbol{R}_{2}\left[\begin{array}{c}
l \\
l \tan \alpha_{v o}^{+} \cos \beta \\
l \tan \alpha_{v o}^{+} \sin \beta
\end{array}\right]+\boldsymbol{A}_{v o}^{+}\right)} \\
\boldsymbol{R}_{2}=\left[\begin{array}{ccc}
\cos \theta & 0 & -\sin \theta \\
0 & 1 & 0 \\
\sin \theta & 0 & \cos \theta
\end{array}\right] \\
\boldsymbol{R}_{3}=\left[\begin{array}{ccc}
\cos \psi & \sin \psi & 0 \\
-\sin \psi & \cos \psi & 0 \\
0 & 0 & 1
\end{array}\right]
\end{array}\right.
$$

式中, $\boldsymbol{R}_{1}$ 计算同式 $(5)$ 。在此基础上, 为得到 $P_{a}$ 所 截得的椭圆形坐标, 应计算 $\beta$ 取不同值时令 $z_{\mathrm{vo}}=0$ 的 $l$ 值, 通过对 (6) 式进行变形,得到(7) 式,进而将 $l$ 带回 (6) 式, 得到 $\left[x_{\mathrm{vo}}, y_{\mathrm{vo}}\right]$, 即为 $\mathrm{VO}_{p}^{+}$下椭圆的坐 标值。

$$
l\left\{z_{\mathrm{vo}}=0\right\}=-\frac{A_{\mathrm{vo}_{z}}^{+} \cos \varphi_{a}-A_{\mathrm{vo}_{y}}^{+} \sin \varphi_{a}}{\left(\sin \varphi_{a} \sin \psi \cos \theta+\cos \varphi_{a} \sin \theta\right)+\tan \alpha_{\mathrm{vo}}^{+}\left(\cos \varphi_{a} \cos \theta \sin \beta-\sin \varphi_{a} \cos \theta \sin \psi-\cos \psi \sin \varphi_{a} \cos \beta\right)}
$$




\section{$2.2 飞$ 行通行权的提出}

下面将上述的三维 VO 法拓展到有人/无人机 系统中, 其关键是要确定各机在避障过程中的飞行 通行权。随着飞机相对位置及速度的更新, 各机的 飞行通行权也相应地更新。

目前, 在有人机编队中常采用的通行法则是

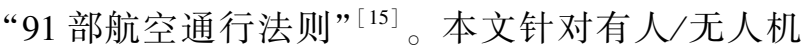
协同系统的特点, 将三维空间中机间的相对位置划 分为右方抵近、左方抵近和相向抵近 3 个区域,并制 定了一套新的通行法则以实现协同飞行,具体如下：

1) 面对外部障碍 (静态障碍和动态障碍), 系 统中所有飞机均没有通行权。

2) 有人机在系统内部具有绝对的领导权,除面 对外部威胁需要躲避以外, 其面对系统内其他无人 机时, 均具有飞行通行权; 当系统内的有人机数量不 止一架时,按照其在系统中的重要程度设定通行权; 另一方面, 系统中各无人机地位均等, 通行权需根据 机间的相对位置确定。

3) 同等地位下,当飞机从右方抵近另一架飞机 时,抵近的飞机具有通行权, 被抵近飞机无通行权; 当飞机从左方抵近另一架飞机时, 情况相反。

4) 同等地位下, 当飞机与另一架飞机相向飞行 时, 2 架飞机均没有通行权。

5）为避免多障碍物下,同一飞机出现避障方向 的矛盾,规定系统中无通行权的飞机统一向飞行方 向的右侧进行避障。

系统中机间的相对位置划分图 4 如下所示。

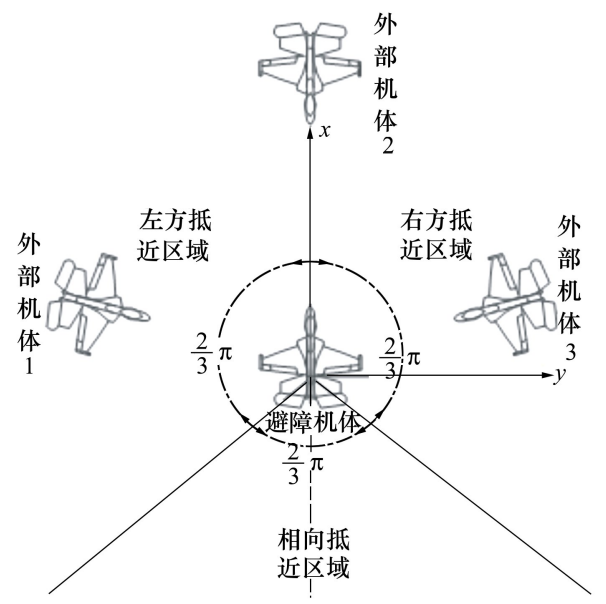

图 4 系统中飞机相对位置划分图

图 4 中坐标系为避障飞机的机体坐标系, 并以 $x o y$ 面为标准面, 当外部机体的航向角相对于避障
机体分别成 $[0,2 \pi / 3],[2 \pi / 3,4 \pi / 3],[4 \pi / 3,2 \pi]$ 角度时,其分别位于避障机体的右方抵近区域、相向 抵近区域和左方抵近区域。特别地, 当外部机体的 航向恰好与相对位置划分的边界重合时, 为保证飞 行安全,应令避障机体优先选择避障。

\section{3 飞行模式的确定}

在上述通行法则的基础上,借鉴选择速度障碍 法的思想 ${ }^{[12]}$, 将三维 VO 法中飞机的飞行模式分为 3 种, 即任务、保持和偏转。

首先, 从运动学角度可对系统中有人机与无人 机进行统一建模。设系统中飞机在飞行中无侧滑， 且发动机推力与飞行速度方向重合, 则飞机在三维 空间内的点质量运动模型,如 (8) 式所示

$$
\left\{\begin{array}{l}
\dot{x}=V_{o} \cos \gamma \cos \chi \\
\dot{y}=V_{o} \cos \gamma \sin \chi \\
\dot{h}=V_{o} \sin \gamma \\
\dot{V}_{o}=g \cdot\left(n_{x}-\sin \gamma\right) \\
\dot{\chi}=g \cdot n_{y} /\left(V_{o} \cos \gamma\right) \\
\dot{\gamma}=g \cdot\left(n_{z}-\cos \gamma\right) / V_{o}
\end{array}\right.
$$

式中: $(x, y, h)$ 表示飞机在地面坐标系下的坐标; $(\gamma, \chi)$ 分别表示飞机的航迹倾角和航向角; $V_{o}$ 表示 飞机地速大小, 且假设避障过程中大小保持不变; $\left(n_{x}, n_{y}, n_{z}\right)$ 分别表示飞机的切向过载、水平法向过 载和垂直法向过载。

针对三维 VO 法, 设飞机的航迹坐标系与机体 坐标系重合, 在 $P_{a}$ 面内, 飞机只通过改变面内偏航 角 $\psi_{p}$ 的方式进行避障, 而面内的切向过载和水平法 向过载保持不变, 则运动方程在 $P_{a}$ 面内可简化为

$$
\left\{\begin{array}{l}
\dot{x}_{p}=V_{o} \cos \psi_{p} \\
\dot{y}_{p}=V_{o} \sin \psi_{p} \\
\dot{h}_{p}=0 \\
\dot{V}_{o}=0 \\
\dot{\psi}_{p}=g \cdot n_{y_{p}} / V_{o} \\
\dot{\theta}_{p}=0
\end{array}\right.
$$

式中,下标 $p$ 表示飞机在 $P_{a}$ 面内的状态量。

其次, 设空间中共有 $N$ 个需要躲避的障碍物, 则 从降低飞机能量损耗的角度出发,利用(10) 式确定 $\psi_{p}$, 即避障角

$$
\left\{\begin{array}{l}
\psi_{p}=\max \left\{\psi_{\mathrm{tep}_{j}}\right\}, j=1,2 \cdots N \\
\psi_{\mathrm{tep}_{j}}=\min \left\{\left|\dot{\psi}_{\max } \Delta t\right|,\left|\varepsilon_{j}\right|\right\}, j=1,2 \cdots N
\end{array}\right.
$$


式中: $\dot{\psi}_{\text {max }}$ 是飞机在平面内允许的最大偏航角速度; $\varepsilon_{j}$ 和 $\psi_{\text {tep } j}$ 分别是飞机面对第 $j$ 个障碍时在平面内向 右侧避障的理论角度和实际角度, 可根据 $\boldsymbol{V}_{o}$ 与截面 内椭圆曲线的几何关系求得。

进而, 最优避障平面 $P_{\text {avo }}$ 可确定如下

$$
P_{\text {avo }}=\left\{P_{a_{i}} \mid \min \left\{\psi_{p_{i}}\right\}, i=1,2 \cdots \pi / \varphi_{o}\right\}
$$

最后, 利用公式 $(5) \sim(6)$ 将 $P_{\text {avo }}$ 内的偏转航迹 反推回惯性坐标系, 得到该飞机的 $(x, y, h)$, 并根据 公式 $(8)$ 解得该时刻的 $(\theta, \psi)$, 显然姿态角的变化 量也满足 $\dot{\psi}_{\text {max }}$ 的约束。

3 种模式的转换关系如图 5 所示, 图中, $d_{\mathrm{avo}}$ 为设 定的开始避障距离。系统完整的避障流程图如图 6 所示。

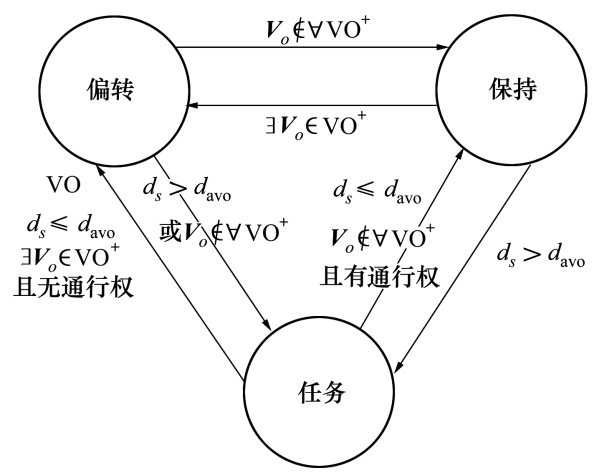

图 53 种飞行模式切换示意图

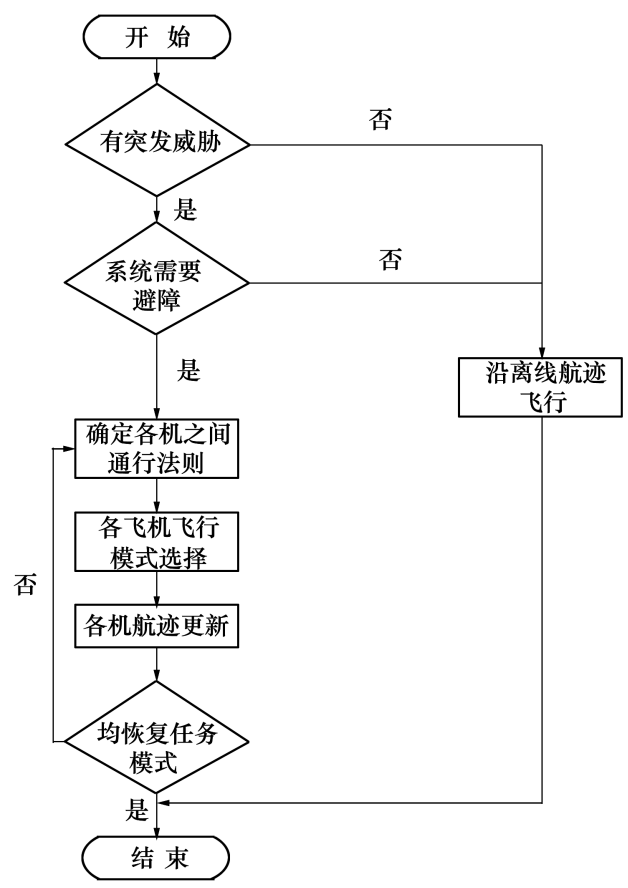

图 6 系统避障流程图

\section{3 仿真校验}

\section{1 避障平面的选择}

假设某机体半径为 $20 \mathrm{~m}$ 的飞机正以 $40 \mathrm{~m} / \mathrm{s}$ 的 速度无侧滑飞行, 其 $\Delta t=1 \mathrm{~s}, d_{\mathrm{avo}}=300 \mathrm{~m}$, 设平面内 最大偏航角速度 $\dot{\psi}_{\text {max }}=0.3 \mathrm{rad} / \mathrm{s}$ 。在某时刻检测到 3 个外部动态威胁, 其相对于本机机体坐标系的状 态量如表 1 所示。

\section{表 1 障碍物状态量表}

\begin{tabular}{cccccc}
\hline $\begin{array}{c}\text { 障碍 } \\
\text { 距离/ 半径/ }\end{array}$ & $\begin{array}{c}\text { 姿态角/ } \\
\text { 标号 }\end{array}$ & $\begin{array}{c}\text { 最大角速 } \\
\mathrm{m}\end{array}$ & $\begin{array}{c}\text { 速度/ } \\
(\mathrm{rad})\end{array}$ & 度/ $\left(\mathrm{rad} \cdot \mathrm{s}^{-1}\right)$ & $\left(\mathrm{m} \cdot \mathrm{s}^{-1}\right)$ \\
\hline 1 & 200 & 36 & $\pi / 4, \pi / 36$ & 0.1 & {$[-20,-10,3]$} \\
2 & 150 & 19 & $\pi / 6, \pi / 4$ & 0.1 & {$[0,-20,20]$} \\
3 & 180 & 17 & $\pi / 7, \pi / 4$ & 0.2 & {$[-15,-30,35]$} \\
\hline
\end{tabular}

表中, 半径为障碍机体半径, 姿态角分别为俯仰 角和偏航角, 最大角速度即 $\dot{\psi}_{\text {max }}$ 。

首先,由表中数据和公式(1) 至(3) 可知,本机 沿当前速度 $\boldsymbol{V}_{o}$ 飞行会与 3 个障碍物均发生碰撞。 另根据 $d_{s}$ 与 $d_{\mathrm{avo}}$ 的大小关系, 可由图 5 确定本机当前 时刻的飞行模式为偏转模式。

其次, 确定避障平面。通过多次仿真可知, $\varphi$ 。 取值过大将无法保证避障的效果，而取值过小则会 明显延长求解的时间, 因而权衡两者关系后, 根据仿 真经验取 $\varphi_{o}=\pi / 6$, 即共离散成 6 个避障平面, 在求 解的侧重点不同时, 该取值可以相应地上下浮动, 不 同 $\varphi$ 。下的仿真对比在本文中不再具体展开。针对 本次仿真, 当 $\varphi_{a}$ 取 $\pi / 6$ 和 $\pi / 2$ 时, 存在不满足式 $\alpha_{v o}^{+}$ $<\pi / 2-\lambda_{a}$ 的情况, 即 $V O_{p}^{+}$的曲线类型存在非椭 圆。具体的避障结果如表 2 和图 7 所示。

飞机为躲避各障碍物而偏转的临界速度矢量终 点在图中已标出, 分别选取左右偏转最大的角度作 为避障平面内的左右避障角, 并进而得到多障碍物 下机体的避障速度, 具体如图 7 中避障速度虚线所 示; 由表中计算结果可知, 当未添加 2.2 节向右避障 的约束时, $P_{\text {avo }}$ 为 $\varphi_{a}=5 \pi / 6$ 的平面, $\psi_{p}=0.2350 \mathrm{rad}$, 向左侧避障可达到最低耗能的避障效果; 而添加向 右避障的约束后, $P_{\text {avo }}$ 为 $\varphi_{a}=2 \pi / 3$ 的平面, 因受 $\psi_{\text {max }}$ 的约束, $\psi_{p}=0.3 \mathrm{rad}$ 。

另外, 在 MATLAB R2013a 中运行上述过程 50 次 (计算机配置为 Intel i7 处理器, 主频 $3.60 \mathrm{GHz}$, 内 存 $32 \mathrm{G}$ ), 得到的平均解算时间为 $0.057 \mathrm{~s}$, 可满足实 时避障的要求,也印证了 $\varphi_{0}$ 取值的合理性。 
表 2 各避障平面的避障结果

\begin{tabular}{ccccccc}
\hline$\varphi_{a}$ & $\lambda_{a} / \mathrm{rad}$ & $\begin{array}{c}\text { 是否存在 } \\
\text { 非椭圆 }\end{array}$ & $\begin{array}{c}\text { 左侧避障 } \\
\text { 角 } / \mathrm{rad}\end{array}$ & $\begin{array}{c}\text { 右侧避障 } \\
\text { 角 } / \mathrm{rad}\end{array}$ & $\begin{array}{c}\text { 左侧避障是否受 } \\
\dot{\psi}_{\text {max }} \text { 约束 }\end{array}$ & $\begin{array}{c}\text { 右侧避障是否受 } \\
\dot{\psi}_{\max } \text { 约束 }\end{array}$ \\
\hline 0 & $0.7854,1.0472,1.1220$ & 否 & 0.5199 & 1.1879 & 是 & 是 \\
$\pi / 6$ & $0.9502,1.4436,1.5136$ & 是 & - & - & - & - \\
$2 \pi / 6$ & $1.2659,1.2867,1.2294$ & 否 & 1.2042 & 1.0072 & 是 & 是 \\
$3 \pi / 6$ & $1.5091,0.9117,0.8801$ & 是 & - & - & - & - \\
$4 \pi / 6$ & $1.1517,0.6756,0.6940$ & 否 & 1.2493 & 0.9960 & 是 & 是 \\
$5 \pi / 6$ & $0.8721,0.7389,0.8034$ & 否 & 0.2350 & 1.1092 & 否 & 是 \\
\hline
\end{tabular}

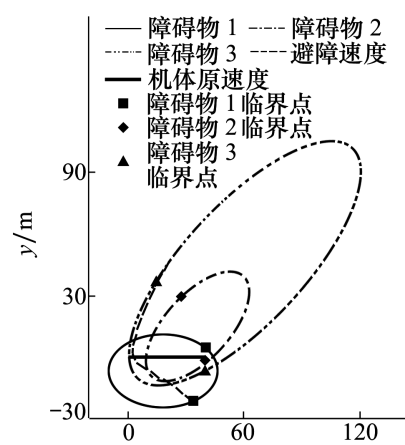

a) $\stackrel{x / m}{\varphi_{a}=0}$

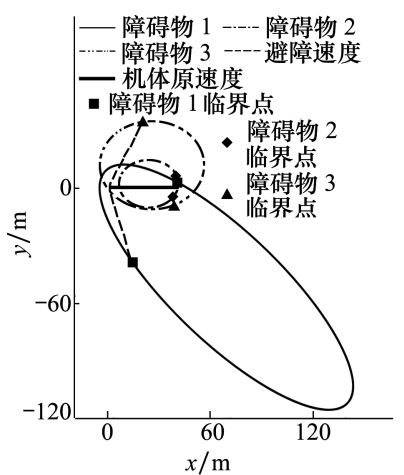

b) $\varphi_{a}=\pi / 3$

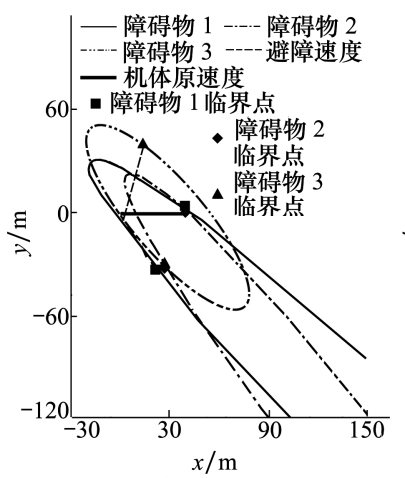

c) $\varphi_{a}=2 \pi / 3$

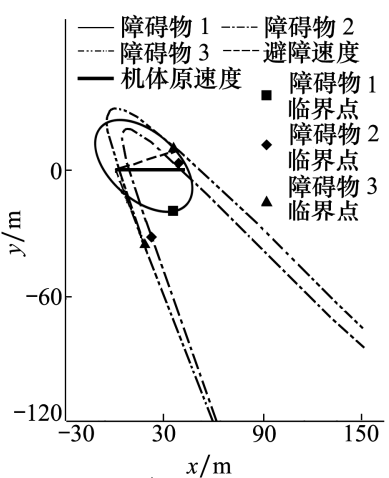

d) $\quad \begin{aligned} & x / \mathrm{m} \\ & \varphi_{a}=5 \pi / 3\end{aligned}$

图 7 各避障平面的避障示意图

\section{2 对具有机动性的障碍物的避障效果检验}

假设某 UAV 在空中无侧滑的飞行, 在某时刻探 测到一外部障碍, 其与 UAV 在该时刻的状态量如表 3 所示 (表中坐标均基于同一惯性坐标系)。

\section{表 3 UAV 与外部障碍状态表}

\begin{tabular}{lccccc}
\hline $\begin{array}{l}\text { 飞行器 } \\
\text { 名称 }\end{array}$ & 位置 & \multicolumn{4}{c}{ 速度/ 姿态角/ 最大角速 半径/ } \\
$\left(\mathrm{m} \cdot \mathrm{s}^{-1}\right)$ & $\mathrm{rad}$ & 度/ $\left(\mathrm{rad} \cdot \mathrm{s}^{-1}\right)$ & $\mathrm{m}$ \\
\hline $\mathrm{UAV}$ & {$[-400,0,0]$} & 30 & $-\pi / 6,2 \pi$ & 0.15 & 25 \\
\hline 外部障碍 $[0,0,400]$ & 50 & $\pi / 3, \pi$ & 0.40 & 25 \\
\hline
\end{tabular}

另外, UAV 在空域中的偏航角幅值无限制, 俯 仰角幅值不超过 $\pi / 3 \mathrm{rad}$ ，由 $\mathrm{UAV}$ 携带的探测设备 可确定 $d_{\mathrm{avo}}=500 \mathrm{~m}, \Delta t=1 \mathrm{~s}$, 且由机体半径可确定 $r$ $=50 \mathrm{~m} ; \mathrm{UAV}$ 与外部障碍的速度大小保持不变, 其 他参数同 3.1 节。

外部障碍相对于 UAV 有绝对通行权, 当其具有 机动性时, 可将其 $\dot{\psi}_{\text {max }}$ 内的某一随机角速度量作为 “扰动项”加人其航迹,其姿态角变化如图 8 所示。 进而, $\mathrm{UAV}$ 分别采用 $V O$ 和 $V O^{+} 2$ 种策略进行避障, 仿真对比结果如图 9 至 11 所示。

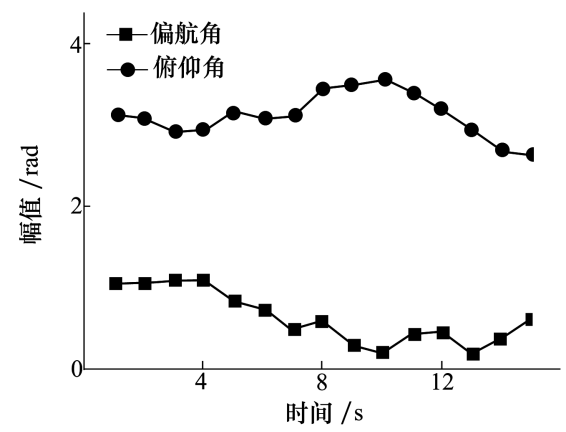

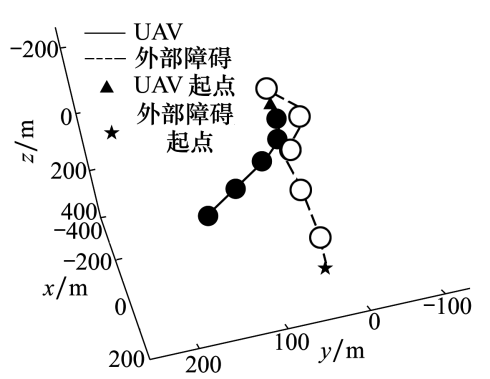

a) 利用 $V O$ 进行动态避障的轨迹图

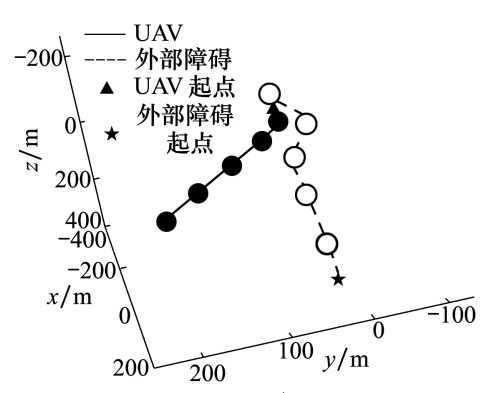

b) 利用 $V O^{+}$进行动态避障的轨迹图 


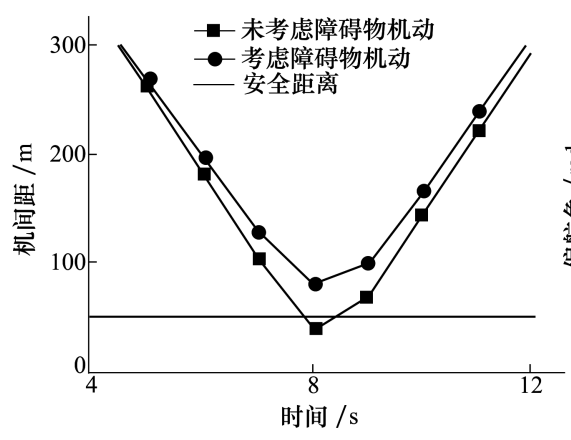

图 102 种策略下飞行器的机间距变化图

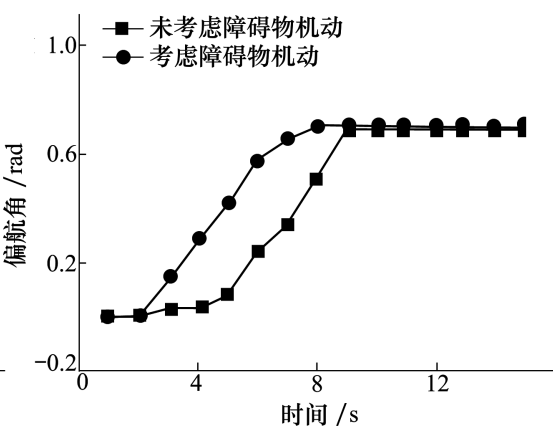

a) 偏航角对比图

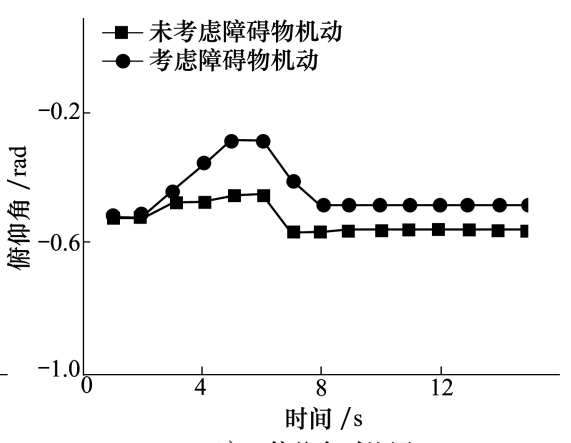

b) 俯仰角对比图
图 9 中实心与空心球体分别表示 UAV 与外部 障碍在不同时刻所处的位置,球体半径即机体半径, 从图中可以看出,利用三维 VO 法可实现 UAV 对外 部障碍的躲避, 但是由于 VO 仅通过前一时刻障碍 物的速度矢量进行计算, 避障效果相比 $\mathrm{VO}^{+}$略差, 可 通过图 10 中局部时间段内的机间距对比图看出; 具 体地, 采取 VO 策略避障, 在探测到障碍物后 7 9 s 时间内, 机间距小于设定的安全距离 $r, \mathrm{UAV}$ 将与外 部障碍发生碰撞; 而采取 $\mathrm{VO}^{+}$策略进行避障时, UAV 与外部障碍的距离始终大于安全距离, 即成功实现 了动态避障。

从图 11 可看出, UAV 利用 2 种策略进行避障 时, 均能满足姿态角幅值和角速度变化约束, 其中利 用 $\mathrm{VO}^{+}$避障时, 偏航角与俯仰角开始变化的时刻均 早于 VO 策略, 特别是偏航角, 其偏转早于 VO 策略 $3 \mathrm{~s}$, 这对同等机动能力的无人机来说是极为关键 的, 这表明 $\mathrm{VO}^{+}$充分考虑了障碍物可能的航迹变化 范围, 为 UAV 的飞行安全提供了重要保障。

\section{3 有人/无人机协同系统避障效果仿真}

假设 1 架有人机与 2 架同构无人机 UAV1、 UAV2 组成协同编队, 按照离线规划航迹在空中飞 行。在某一时刻检测到一破坏性武器(外部障碍) 正朝编队飞来, 此时各飞行器的状态量如表 4 所示 (基于同一惯性坐标系)。

\section{表 4 发现外部障碍时刻各飞行器状态表}

\begin{tabular}{cccccc}
\hline 飞行器 & 位置 & 速度/ & 姿态 & \multicolumn{2}{c}{ 最大角速度半径/ } \\
名称 & $\left(\mathrm{m} \cdot \mathrm{s}^{-1}\right)$ & 角 $/ \mathrm{rad}$ & $/\left(\mathrm{rad} \cdot \mathrm{s}^{-1}\right)$ & $\mathrm{m}$ \\
\hline $\mathrm{UAV} 1$ & {$[-400,200,0]$} & 30 & $-\pi / 6,5 \pi / 3$ & 0.20 & 25 \\
有人机 $[-400,200,0]$ & 30 & $-\pi / 6,2 \pi$ & 0.20 & 25 \\
$\mathrm{UAV} 2[-400,-200,0]$ & 36 & $-\pi / 6, \pi / 6$ & 0.20 & 25 \\
外部障碍 $[0,0,400]$ & 40 & $\pi / 3, \pi$ & 0.40 & 25 \\
\hline
\end{tabular}

图 112 种策略下 UAV 姿态角变化图

设各飞机携带同性能的探测设备, 与 3.2 节保 持一致, 即 $d_{\mathrm{avo}}$ 以及 $\Delta t$ 的取值不变, 且有 $r=50 \mathrm{~m}$, 其 他参数同 3.1 节; 各飞机的初始飞行模式均为 “任 务”, 并设该模式下各机均保持当前姿态匀速飞行。 在不采取三维 VO 法进行避障的情况下, 各飞行器 的航迹图和机间距变化图如图 12 至 13 所示。

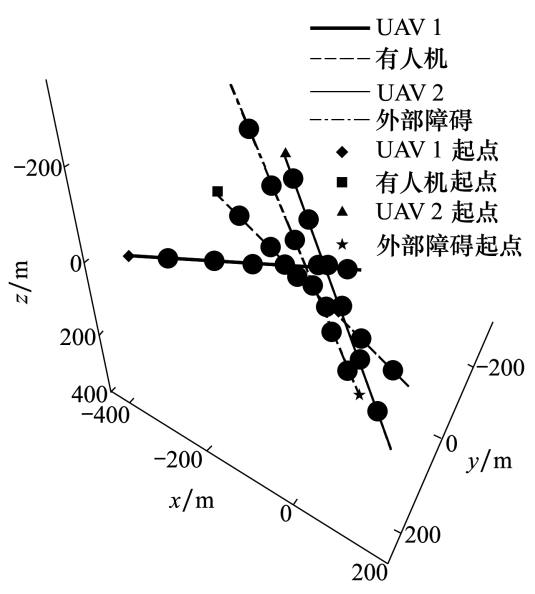

图 12 任务模式下编队航迹变化图

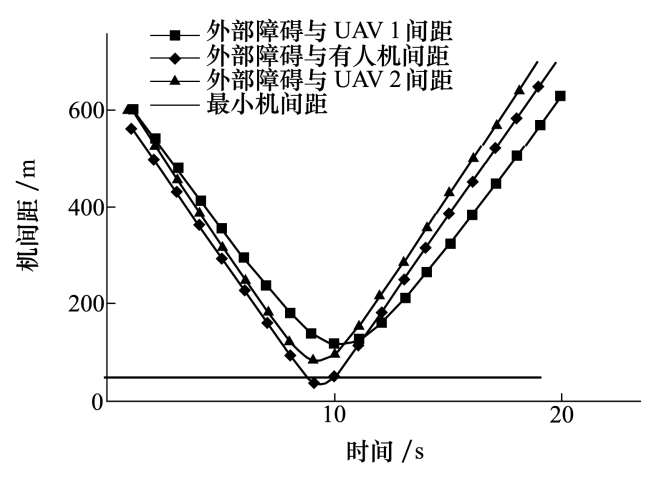

图 13 任务模式下各机与外部障碍的间距变化图 
飞机每隔 $3 \mathrm{~s}$ 所处的位置, 球体半径即机体半径。 由图 13 中可以看出, 编队若保持 “任务” 模式继续 飞行, 有人机会与外部障碍在 $9 \sim 10 \mathrm{~s}$ 时发生碰撞, 若仅为了躲避外部障碍而改变有人机的航迹,则易 导致其与编队中其他 UAV 发生碰撞, 因此应采用文 中所述方法对编队采取动态避障。

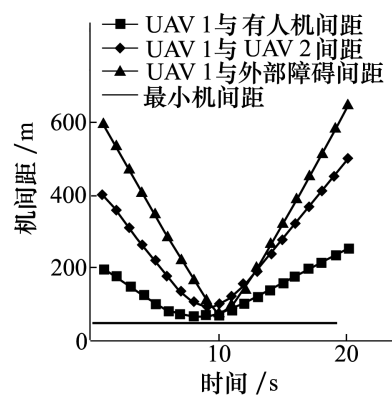

a) UAV1 与其他飞机的间距

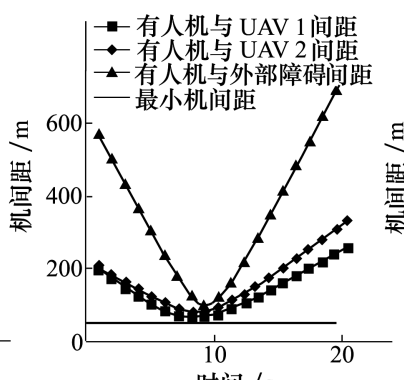

b） 有人机与其他飞机的间距

为校验通行法则的优先级次序, 设外部障碍未 发生主动机动, 且动态避障过程中各飞行器速度大 小保持恒定, 姿态角幅值及 $\varphi_{0}$ 与 3.2 节保持一致。 截取当前时刻之后的 $20 \mathrm{~s}$ 区间进行仿真分析,结果 如图 14 至 17 ,其中每一离散时刻的平均解算耗时 为 $0.079 \mathrm{~s}$, 远小于 $\Delta t$ 。

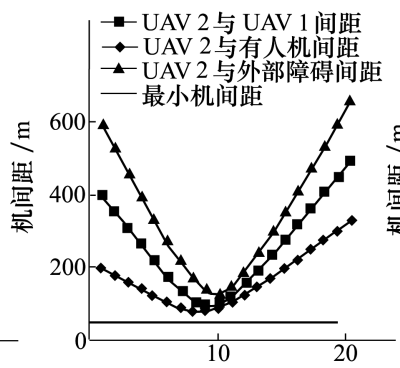

时间 /s

c） UAV2 与其他飞机间距

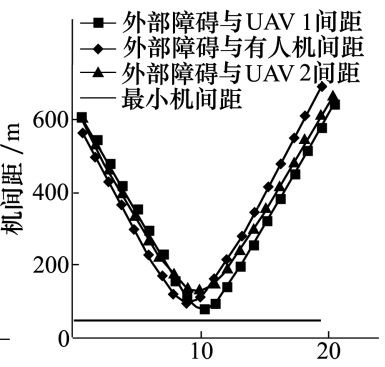

时间 /s

d） 外部障碍与其他飞机间距

图 14 三维 VO 法避障航迹的机间距变化图

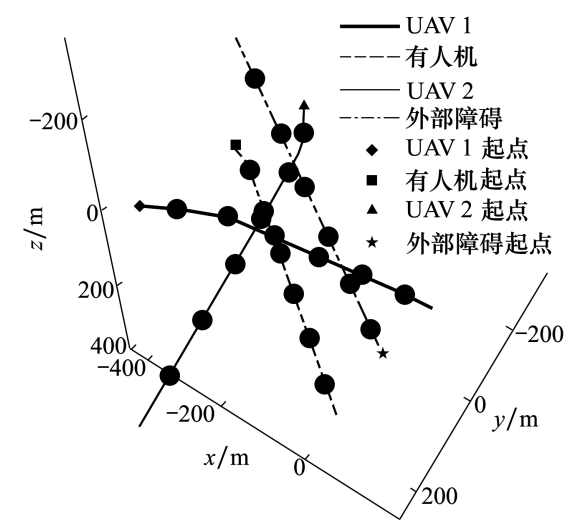

图 15 三维 VO 法规划航迹的变化图

由图 14 可知,三维 VO 法使各飞行器的间距始

终大于安全距离,即能够实现动态避障; 该模型中, 外部障碍的优先级高于有人机, 同时有人机高于 $\mathrm{UAV}$;由图 15 可知,外部障碍未发生机动,有人机为 躲避外部障碍做出相应机动, 2 架同等地位的 UAV 机动最大; 结合图 16 的姿态角变化图可校验这一结 论, 从该图中可以看出, 各机的姿态角变化均满足幅 值、角速度变化以及向右偏转避障的约束, 未出现短 时间内的大幅度波动, 利于系统飞行的稳定性; 另 外,图 17 中, 飞行模式 $1 、 2 、 3$ 分别代表“任务”、“偏 转” 和“保持” 模式, 各机的机动时刻点与该图所示 的飞行模式变化节点一一对应, 且有人机由于通行 的优先级高而早于 2 架 UAV 进人 “模式 3”, 从而可 进一步校验通行法则的正确性和有效性。

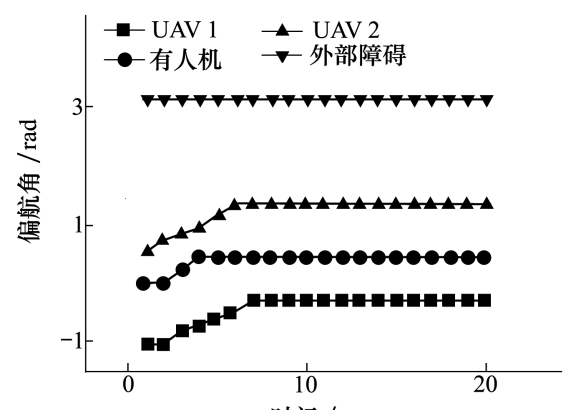

时间 /s

a) 各飞行器的偏航角对比图

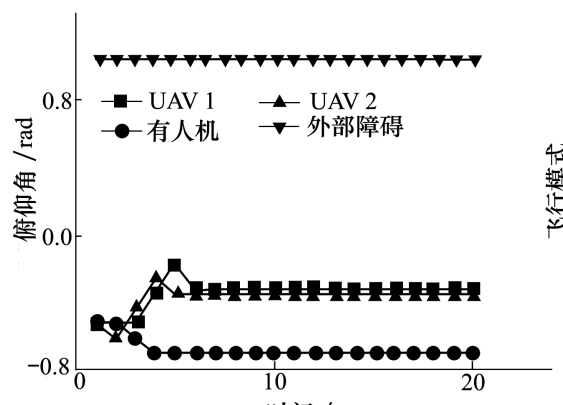

时间 /s

b) 各飞行器的俯仰角对比图

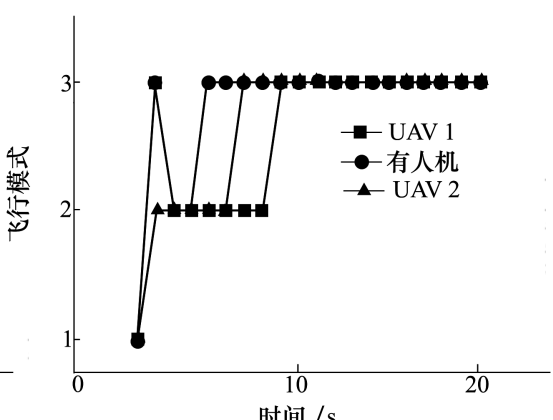

时间 /s

图 16 各飞行器的姿态角变化图

图 17 三维 VO 法规划的 飞行模式变化图 


\section{4 结 论}

本文利用改进的速度障碍法, 对有人无人机协 同系统三维实时避障问题进行了研究。分析和仿真 结果表明:

1) 传统速度障碍物法适用于多障碍物避障, 且 原理简单, 计算效率高, 可拓展到三维异构系统的避 障中。

2) 利用考虑障碍物机动的三维速度障碍模型 进行动态避障, 能有效提高避障水平, 保证避障机体
的飞行安全。

3) 引人避障平面, 将三维避障问题进行降维, 通过避障角的比较对避障平面进行选择, 有效提高 了计算效率,适用于实时避障。

4) 有人无人机协同系统避障的通行法则为系 统避障提供指导, 有效保证了有人机的绝对领导权 和安全稳定性,利于实现协同飞行。

考虑避障平面内不同曲线类型的处理方式、加 人障碍物速度机动并将所提方法利用飞行试验进行 验证是下一步值得研究的内容。

\section{参考文献:}

[1] 申超,李磊,吴洋,等. 美国空中有人无人自主协同作战能力发展研究 $[\mathrm{J}]$. 战术导弹技术, 2018, 1: 16-21

SHEN Chao, LI Lei, WU Yang, et al. Research on the Capability of the U.S. Manned/Unmanned Autonomous Collaborative Operations [J]. Tactical Missile Technology, 2018, 1:16-21 (in Chinese)

[2] HUMPHREYSY C J, COBBZ R G, JACQUESX D R, et al. Optimal Mission Paths for the Uninhabited Loyal Wingman[C] // 16th AIAA/ISSMO Multidisciplinary Analysis and Optimization Conference, Reston, 2015:2792-2802

[3] The Department of Defense of USA. Unmanned System Integrated Roadmap[R]. FY2013-2038, 2014

[4] 贾高伟,侯中喜. 美军有/无人机协同作战研究现状与分析 $[\mathrm{J}]$. 国防科技. 2017, 38(6) : 57-59

JIA Gaowei, HOU Zhongxi. The Analysis and Current Situation about the United States Military Manned/Unmanned Aerial Vehicle $[\mathrm{J}]$. National Defense Science \& Technology, 2017, 38(6) : 57-59 (in Chinese)

[5] ChaKravarthy A, GHOSE D. Obstacle Avoidance in a Dynamic Environment: a Collision Cone Approach[J]. IEEE Trans on Systems, Man and Cybernetics, Part A: Systems and Humans, 1998, 28(5) : 562-574

[6] SCHMITT L, FICHTER W. Collision-Avoidance Framework for Small Fixed-Wing Unmanned Aerial Vehicles[J]. Journal of Guidance, Control, and Dynamics, 2014, 37(4) : 1323-1329

[7] ANDRIY A, ALEKSANDR V, OLEKSANDR V, et al. Improvement of the Anti Collision Method 'Velocity Obstacle' by Taking into Consideration the Pyamics of an Operating Vessel[ J]. Eastern-Earopean Journal of Enterprise Technologies, 2019, 6(3) : 14-19

[8] KLUGE B, PRASSLER E. Recursive Probabilistic Velocity Obstacles for Reflective Navigation[J]. Field and Service Robotics, $2006(24): 71-79$

[9] MUJUMDAR A, PADHI R. Reactive Collision Avoidance of Using Nonlinear Geometric and Differential Geometric Guidance [J]. Journal of Guidance, Control, and Dynamics, 2012, 34(1) : 303-311

[10] FIORINI P, SHILLER Z. Motion Planning in Dynamic Environments Using Velocity Obstacles $[\mathrm{J}]$. International Journal of Robotics Research, 1998, 17(7): 760-772

[11] 杨秀霞, 周硙硙, 张毅. 基于速度障碍圆弧法的 UAV 自主避障规划研究 $[\mathrm{J}]$. 系统工程与电子技术, 2017, 39(1)： 168-177

YANG Xiuxia, ZHOU Weiwei, ZHANG Yi. Automatic Obstacle Avoidance Planning for UAV Based on Velocity Obstacle Arc $\operatorname{Method}[\mathrm{J}]$. Systems Engineering and Electronics, 2017, 39(1) : 168-177 (in Chinese)

[12] JENIE Y I, VAN KAMPEN E J, DE VISSER C C, et al. Selective Velocity Obstacle Method for Deconflicting Maneuvers Applied to Unmanned Aerial Vehicles[J]. Journal of Guidance, Control, and Dynamics, 2015, 38(6) : 1140-1145

[13] SNAPE J, VAN DEN BERG J, GUY S. The Hybrid Reciprocal Velocity Obstacle[J]. IEEE Trans on Robotics, 2011, 4: 696-706

[14] CHARKRavarthy A, GHOS D. Generalization of the Collision Cone Approach for Motion Safety in 3-D Environments [J]. 
Autonomous Robots, 2012, 32(3) : 243-266

[15] AMDT. 14 CFR § 91.113-Right-of-Way rules: Except Water Operations[R]. National Archives and Records Administration's Office of the Federal Register, 91282, 69 FR 44880, 2004

\title{
Real-Time Obstacle Avoidance for Manned/Unmanned Aircraft Cooperative System Based on Improved Velocity Obstacle Method
}

\author{
LI Yue ${ }^{1}$, HAN Wei ${ }^{1}$, CHEN Qingyang ${ }^{2}$, ZHANG Yong ${ }^{1}$ \\ 1.College of Basic Science for Aviation, Naval Aviation University, Yantai 264001, China; \\ 2.College of Aeronautics and Astronautics, National University of Defense Technology, Changsha 410073, China)
}

\begin{abstract}
To adapt the autonomous level of agents in current, and to perform the advantages of multi-agent in air combat, the form of manned/unmanned aircraft cooperative system has gradually become a hot topic. To solve the issue of three-dimensional (3D) real-time obstacle avoidance, the 3D maneuvering obstacle model is established firstly based on the traditional velocity obstacle method. Then the flight mode is selected and the optimal obstacle avoidance plane is determined by setting the Right-of-way rules when the system encountering obstacles. Finally, the difference of obstacle avoidance plane, the feasibility of avoiding maneuvering obstacle and the effectiveness of obstacle avoidance of cooperative system are verified by several flight simulations. The results show that the proposed method can realize the avoidance of 3D maneuvering obstacle for manned/unmanned aircraft cooperative system safely and efficiently.
\end{abstract}

Keywords: manned/unmanned aircraft; velocity obstacle method; three-dimensional space; obstacle avoidance plane; real-time avoidance; flight simulation; cooperative system 